\title{
Review of Different Inpainting Algorithms
}

\author{
Pritika Patel \\ Computer sci. \& Engg. \\ Parul Institute of Engg. \\ \&Technology, \\ Vadodara, Gujarat.
}

\author{
Ankit Prajapati \\ Computer Engg. \\ Parul Institute of Technology, \\ Vadodara, Gujarat.
}

\author{
Shailendra Mishra \\ Asst. Prof. in Computer Engg. \\ Parul Institute of Technology, \\ Vadodara, Gujarat.
}

\begin{abstract}
Image inpainting was historically done manually by painters for removing defect from paintings and photographs. Fill the region of missing information from a signal using surrounding information and re-form signal is the basic work of inpainting algorithms. Here in this paper we have studied and reviewed many different algorithms present for doing image inpainting and explain their approach. We have briefly explain some algorithms for video inpainting applications. This paper contain work done in the field of image inpainting and guide newcomers who are willing to work in image inpainting field.
\end{abstract}

\section{Keywords}

Image inpainting, Texture Synthesis, PDE

\section{INTRODUCTION}

"Inpainting is the process of reconstructing lost or deteriorated parts of images and videos. For instance, in the case of a valuable painting, this task would be carried out by a skilled image restoration artist. In the digital world, inpainting (also known as image interpolation or video interpolation) refers to the application of sophisticated algorithms to replace lost or corrupted parts of the image data (mainly small regions or to remove little defects."[29].

There are many applications of image inpainting. It can be used in cinema and photography for "restoration", for removing effects like scratches, dust spot from images (called deterioration). It can also be used for removing some object from image or removing red eye removal. Image denoising is famous problem in image processing field. Image inpainting and image denoising are not same. Below figure shows the difference between both.
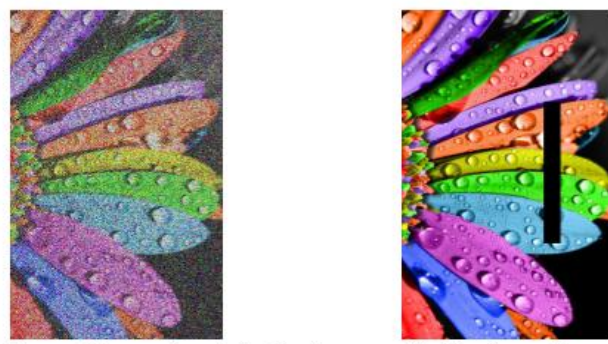

Noisy Image(Image denoising) Image with black spot(image inpainti

Figure 1. Difference between image denoising and image inpainting
Image inpainting can use for privacy protection. Many video obfuscation techniques are available $[28,13]$ in which one can remove objects from given video frame. Below diagram shows one such example presented in [18].
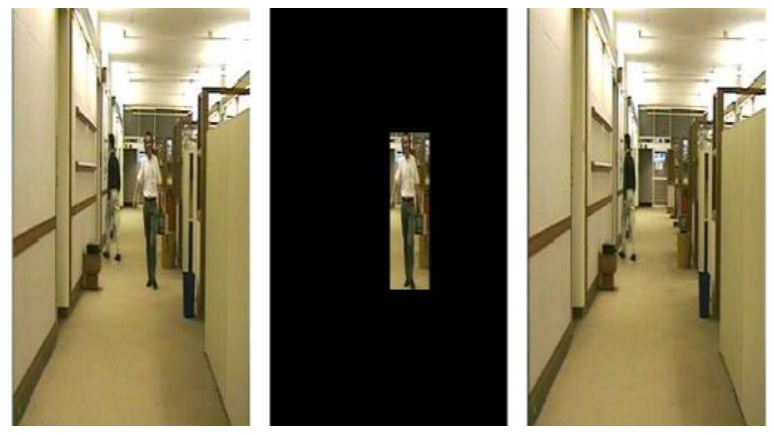

Figure 2. Image inpainting application for video presented in [18]

Paper is organized as follow. Section 2 contain different inpainting algorithms. In section 3 we have given a brief idea of video image inpainting, how to check or evaluate quality of inpainted image is explained in section 4, and here in section 5 we have briefly explain some of the recent work in this field. Finally section 6 contain conclusion and future work.

\section{INPAINTING ALGORITHMS}

Image inpainting algorithms can be classified into different categories like texture synthesis based image inpainting, Exemplar and search based image inpainting, PDE (Partial Differential Equation) based inpainting, Fast semiautomatic inpainting and hybrid inpainting. Here in this section we have explained all of these inpainting methods.

\section{Texture synthesis based image inpainting}

In this method, holes are filled by sampling and copying neighboring pixels[2,7,9]. Main difference between different texture based algorithms is how they maintain continuity between hole's pixel and original image pixels. This method is only work for selected number of images, not with all. Yamauchi et.al presented algorithm which generate texture under different brightness condition and work for multi resolution [11].Bergen proposed algorithm where matching texture is synthesized from target texture [7].Fast synthesizing algorithm presented in [2], uses image quilting (stitching small patches of existing images). 
All texture based methods are different in terms of their capacity to generate texture with different color, intensity, gradient and statistical characteristics. Texture synthesis based inpainting method not perform well for natural images. These methods not handle edges and boundaries well. In some cases user need to enter which texture to replace with which texture. So these methods are used for small area of inpainting.

\section{Exemplar and search based image inpainting}

This method is very effective and use Isophote driven Inpainting and texture synthesis proposed by Criminisi et.al [1]. In this algorithm priority based mechanism is used to determine order of region filling. This method work very good for large number of images. It uses good texture and structure replication. Problems with this method is, curved structure are not handle properly and biasing in due to incorrect selection of patches.

In [6] Fang et.al presented one algorithm by combining direction measure with texture synthesis based technique presented in [4]. In algorithm presented by drori etal[12] to find unknown region, iterative approximation used. Till now to fill the hole in image one use same image pixels only but hays et.al [14] gives the concept of using millions of images as the database for filling hole. The nearest and perfect match for the image is obtain by database searching. Below we have shown figure adopted from [14] which given an idea how searching technique work. Position blending process can also be used with searching technique to fill hole.

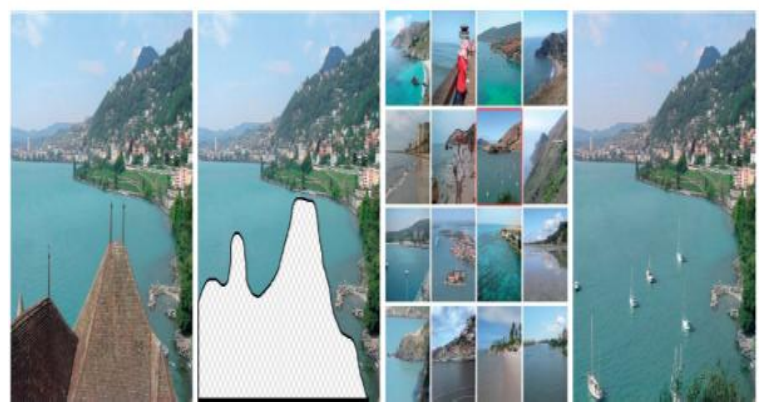

Figure 3. Search based inpainting[14]

\section{PDE based inpainting}

First PDE base approach given by Bertalmio et.al [21]. It uses the concept of isophotes (linear edges of surrounding area) and diffusion process. Main problem with this method is that due to blurring effect of diffusion process replication of large texture is not perform well. Pixels on edges are also not handled properly. TV (Total Vibrational) model is proposed by Chan and Shen which uses anisotropic diffusion and Euler-Lagrange equation. From TV model, another algorithm presented based on CDD (Curvature Driven Diffusion model) which include curvature information of the isophotes. Another PDE based technique known as vector valued regularization under anisotropic diffusion framework presented by Tschumperle et.al [8].

\section{Fast semi-automatic inpainting}

A two step process is proposed by Jian etal[15] called inpainting with Structure propagation. A fast inpainting method is proposed by Oliviera etal[23] which do inpainting using iterative convolving inpainting region with diffusion kernel. Another method which uses FMM (Fast Marching Method) for image information propagation. This method is not suitable for images with large size holes as for edge region no specific method used.

\section{Hybrid Inpainting}

In this method, PDE and texture synthesis based inpainting methods are combined for filling holes. Here main goal is to decompose image into texture and structure region. Then corresponding regions are filled by texture synthesis and edge propagating algorithms respectively [22]. It require more computational time for large holes. Structure completion through segmentation based inpainting technique is found in [16]. Segmentation algorithm used in this method is presented in [31].

\section{VIDEO INPAINTING}

Main applications of video inpainting is in video modification for privacy protection [28], video error concealment in video transmission, video stabilization, multimedia editing and visualization. Normal method to do video inpainting is to treat video as number of frames and applying image inpainting in all frames. Problem with this method is, one cannot utilize the temporal redundancy present in video frames. First method for video which is PDE based was proposed by Bertalmio et.al and uses Navier-Stokes equation. It does not consider temporal redundancy. A space time patch model was proposed by Cheung et.al which was based on probabilistic learning with applications to inpainting. Video inpainting algorithm is proposed by Patwardhan et al.[19] which was a modified version of priority based exemplar approach proposed in [1]. A scheme for video has been proposed in [32] which is based on motion layer estimation. The method followed by motion compensation and texture completion. Same kind of algorithm for perspective camera under constrained motion is proposed recently. Object-based inpainting system which utilizes a user supported segmentation to inpaint holes in foreground regions is proposed by Jiaya et.al [17] which contain cyclic motions. Similar technique which use nonparametric texture synthesis coupled with graph cuts and mean shift tracking to bounds search space has been proposed [30].

\section{PARAMETERS FOR INPAINTING ALGORITHM EVALUATION}

For find out or compare the quality of different inpainting algorithms, not much research has been done. Initially algorithms were compared based on their capability to handle big fill areas, how good algorithm in curved structures, texture replication capability, time taken and algorithm work for how many images etc.[21]. Some time images are converted in RGB to compare their visual quality. In [8] they have used PSNR (Pick signal to noise 
ratio) and MSE (Mean squared error) for comparing inpainting algorithms. Some time domain of original image is also considered for comparison. Below figure adopted from [27] shows domain shape based quality checking. Nowadays parameter used to check the quality of inpainting algorithm is to check how inpainted image closer to original image. Ardi [25] very recently analyze the visual salience map generated by a computational vision model [20]). They relate it to the perceptual quality of image inpainting.
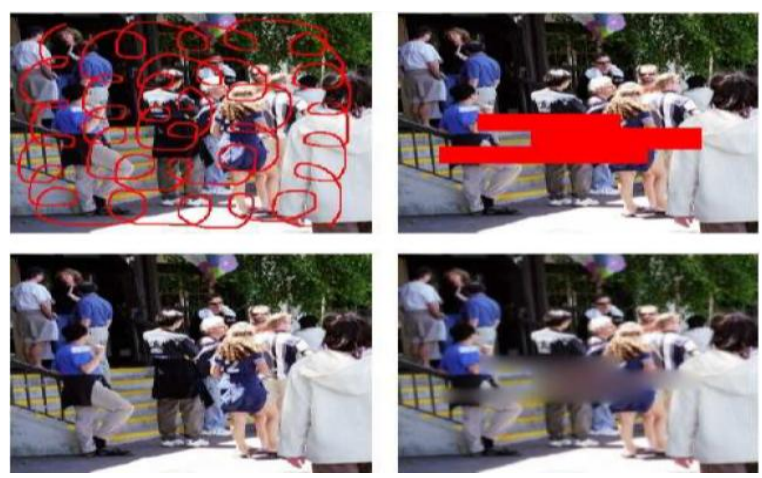

Figure 4. Domain shape based quality[27]

\section{RECENT WORK}

We have studied some recent research paper in detain. In this section we have describe all that and show the results achieved by these papers.

In [24] authors presented TV based texture inpainting algorithm which simultaneously inpaints structures and textures of damaged images. Their algorithm decomposes image into two parts. They inpaint the cartoon image part firstly using boundary reconstruction. Then they use texture synthesis to texture image part guided by boundary reconstruction. Their algorithm gives good results for texture with complex structure. Below we have shown the result produce by their algorithm. They have compare it with M.Bertalmio(indicated "[1]" in figure) and with crimini.

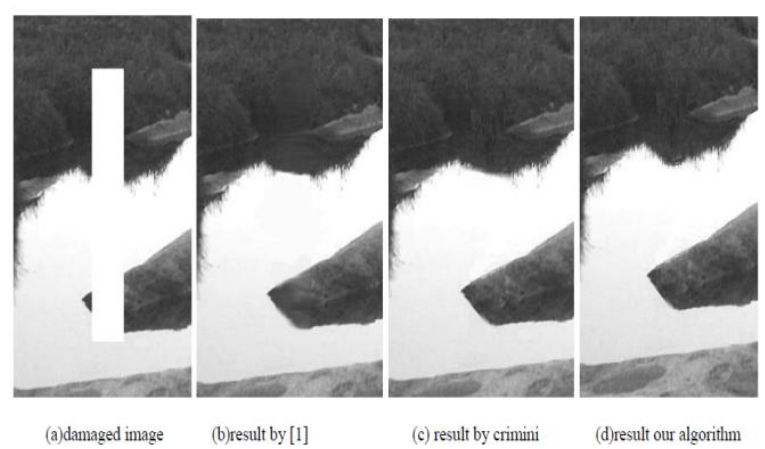

Figure 5. Result and comparison of algorithm presented in [24]
Inpainting algorithm based on directional mean filters is presented in [10]. They have proposed iterative algorithm. The algorithm works as follow. "First iteration calculate median value of known pixels' in each direction and then damaged pixel is replaced by the median of the obtained values. In latter iterations, median of all pixels' values in each direction is calculated then median of obtained values is copied in place of the damaged pixel. This algorithm is fast and provides adequate results in sharp edges regions." Below figure shows the results and comparison of proposed method.
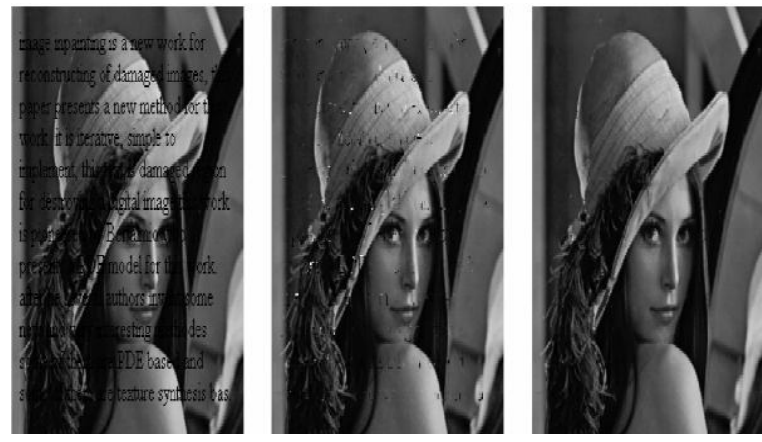

Figure 6. Result and comparison for algorithm presented in [10]

Fast exemplar based algorithm presented in [3]. Their approach can inpaint large regions. Algorithm presented in paper is "is to find examples (i.e. patches) from the image and replace the lost data with it". Their technique can be used in restoring old photographs or damaged film. It can also remove superimposed text like dates, subtitles etc.; or even entire objects from the image like microphones or wires to produce special effects. Result is given below. In figure "[3]" represent A. Criminisi, P. Perez, and K. Toyama, "Region Filling and Object Removal by Exemplar- Based Image Inpainting," IEEE Transactions on Image Processing, 13(9), 1200-1212, 2004.

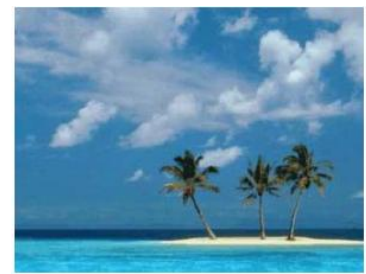

(a)

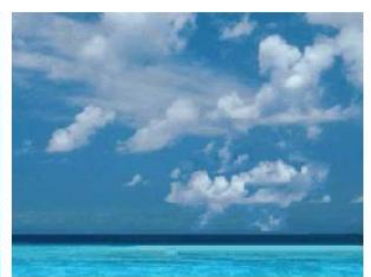

(b)

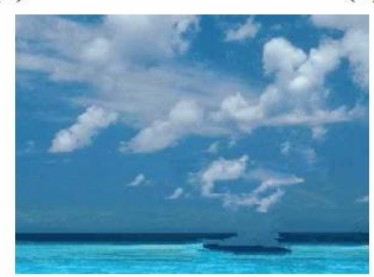

(c)

Figure 7. Result and comparison of algorithm presented in [3] 
In [5] algorithm based on multiscale salient structure propagation is proposed. The brief of algorithm is "approach consists of four stages, namely, (1) detection of salient structure(s), (2) inpainting of salient structure(s), (3) inpainting of surrounding areas of salient structure(s) by modified ant colony optimization (ACO), and (4) inpainting of remaining missing regions." Below we have shown result of proposed algorithm. In below figure "[b]" is M. Bertalmío, "[e]" is Y. Chen, Q. Luan, H. Li, and Oscar $\mathrm{Au}$, "Sketch-guided texture-based image inpainting," and "[gs]" is J. Shen, X. Jin, C. Zhou, and C. C. L. Wang, "Gradient based image completion by solving the Poisson equation,".

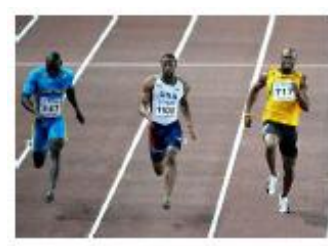

(a)

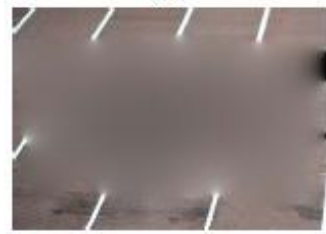

(c)

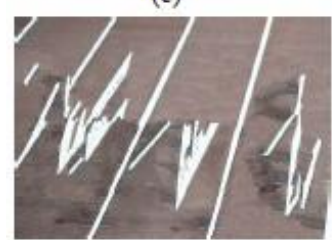

(e)

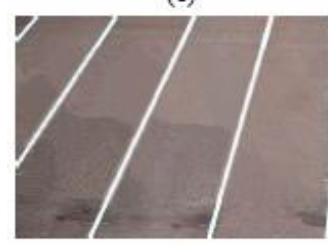

(g)

Figure 8. Result and comparison for algorithm presented in [5]

In [26] new image inpainting method based on exemplarbased image inpainting idea by Curvature-Driven Diffusion (CDD) model is presented. Their method can effectively prevent the "garbage" from producing during the process of inpainting, which is a common problem faced in other methods. Results are below. Here "[7]" is A. Criminisi, P. Pérez, K. Toyama. Region filling and object removal by exemplar-based image inpainting and "[10]" is H. J. Peng, W. X. Hou, N. S. Gong. An improved exemplar-based inpainting method for object remove.
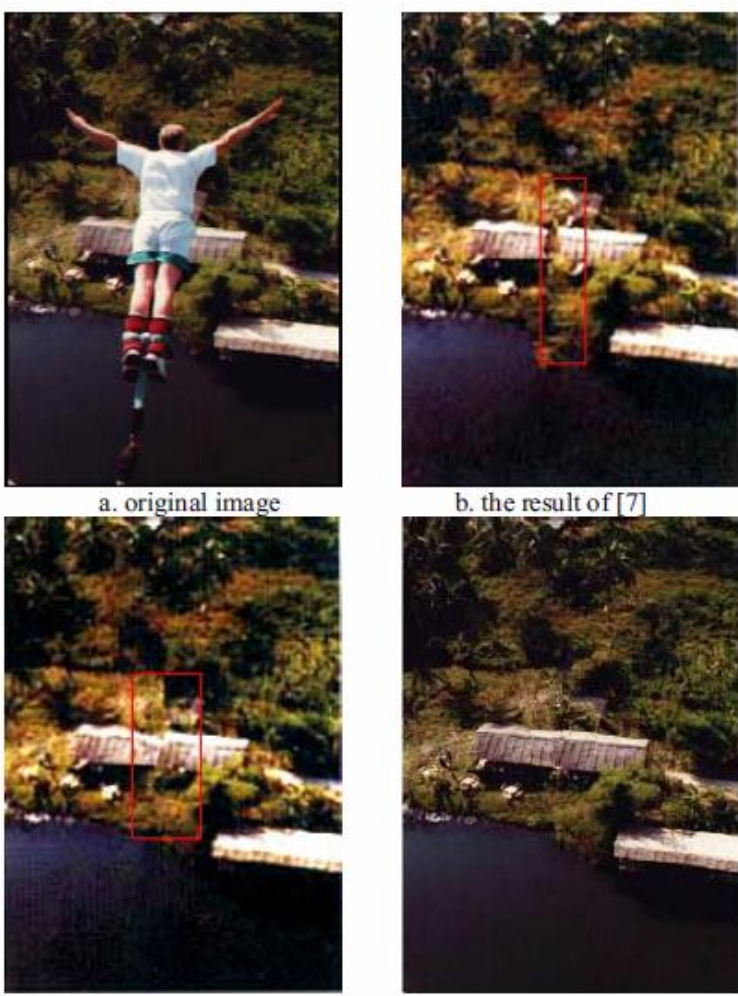

b. the result of [7]

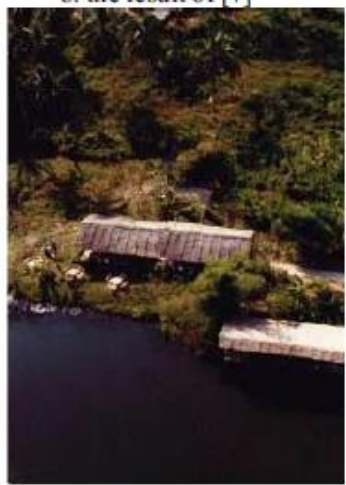

Figure 9. Result and comparison of algorithm presenter in [26]

\section{CONCLUSION AND FUTURE WORK}

Image inpainting is recently very important research area in the field of image processing. Still many work on images and on videos can be done. The applications as we have listed are many of inpainting algorithms. In future we would like to implement all recent algorithm presented by us and would like to compare them. We would like to improve those algorithm if possible and would like to propose our new inpainting algorithm.

\section{REFERENCES}

[1] Criminisi, Patrick Perez, and Kentaro Toyama. Region filling and object removal by exemplar-based inpainting. IEEE Transactions on Image Processing, 13(9):1200\{1212, September 2004.

[2] Efros and W.T. Freeman. Image quilting for texture synthesis and transfer. In Proceedings of ACM Conf. Comp. Graphics (SIGGRAPH), pages 341\{346, August 2001

[3] Anupam , Pulkit Goyal , Sapan Diwakar, "Fast and Enhanced Algorithm for Exemplar Based Image Inpainting", 2010 Fourth Pacific-Rim Symposium on Image and Video Technology.

[4] C.W. Fang and J.J.J. Lien. Fast image replacement using multi-resolution ap- proach.

[5] Cheng-Shian Lin, Jin-Jang Leou, "Image Inpainting Using Multiscale Salient Structure Propagation”, 2011 
International Conference on Multimedia and Signal Processing.

[6] Chih-Wei Fang and Jenn-Jier James Lien. Rapid image completion system using multi-resolution patch-based directional and non-directional approaches. IEEE Transactions on Image Processing, 18(11), 2009.

[7] D.J. Heeger and J.R. Bergen. Pyramid-based texture analysis/synthesis. In Proceedings of ACM Conf. Comp. Graphics (SIGGRAPH), volume 29, pages 229\{233, Los Angeles, CA, 1995.

[8] David Tschumperl and Richard Deriche). Vectorvalued image regularization with pde's : A common framework for different applications. IEEE Transactions on Pattern Analysis and Machine Intelligence, 27(4):506\{517, 2005.

[9] H. Igehy and L. Pereira. Image replacement through texture synthesis. In Proceedings of International Conference on Image Processing (ICIP), volume III, pages 186\{190, 1997.

[10] H. Noori , Saeid Saryazdi , "Image Inpainting Using Directional Median Filters", 2010 International Conference on Computational Intelligence and Communication Networks.

[11]photographs. ACM Transactions on Hitoshi Yamauchi, JÄorg Haber, and Hans-Peter Seidel. Image restoration using multiresolution texture synthesis and image inpainting. In Computer Graphics International (CGI 2003), pages $120\{125$, Tokyo, Japan, July 2003. IEEE.

[12] Iddo Drori, Daniel Cohen-Or, and Hezy Yeshurun. Fragment - based image completion. In Proceedings of ACM Conf. Comp. Graphics (SIGGRAPH), volume 22, pages $303\{312$, July 2003.

[13] J. Wickramasuriya, M. Datt, S. Mehrotra, and N. Venkatasubramanian. Privacy protecting data collection in media spaces. In ACM International Conference on Multimedia, pages $48\{55$, New GRAPH), volume 24, pages $861\{868$, July York, NY, October 2004.

[14] James Hays and Alexei A Efros. Scene completion using millions of Graphics (SIGGRAPH 2007), 26(3), 2007.

[15] Jian Sun, Lu Yuan, Jiaya Jia, and Heung-Yeung Shum. Image completion with structure propagation. In Proceedings of ACM Conf. Comp. Graphics (SIG2005.

[16]Jiaya Jia and Chi keung Tang. Inference of segmented color and texture description by tensor voting. IEEE Transactions Pattern Analysis and Machine Intelligence (PAMI), 26(6):771 \{786, June 2004.

[17] Jiaya Jia, Yu-Wing Tai, Tai-Pang Wu, and Chi-Keung Tang. Video repairing under variable illumination using cyclic motions. In IEEE Transactions on Pattern Analysis and Machine Intelligence (PAMI), volume 28, pages 832\{839, May 2006.

[18] K. P. Karmann and A. Brandt. Moving object recognition using an adaptive memory background. In V. Cappellini, editor, Time-Varying Image Processing and Moving Object Recognition, pages 289\{307. Elsevier Science Publishers, 2nd edition, 1990.

[19] K. Patwardhan, G. Sapiro, and M. Bertalmio. Video inpainting of occluded and occluding objects. In Proceedings of IEEE International Conference on Image Processing (ICIP), volume 2, pages 69\{72, 2005 .

[20] L. Itti, C. Koch, and E. Niebur. A mode of saliencybased visual attention for rapid scene analysis. IEEE Transactions on Pattern Analysis and Machine Intelligence, 20(11):1254\{1259, November 1998.

[21] M. Bertalmio, G. Sapiro, V. Caselles, and C. Ballester. Image inpainting. In Proceedings of ACM Conf. Comp. Graphics (SIGGRAPH), pages $417\{424$, New Orleans, USA, July 2000.

[22] M. Elad, J.-L Starck, D. Donoho, and P. Querre. Simultaneous cartoon and texture image in-painting using morphological component analysis (mca). Applied and Computational Harmonic Analysis, 2005. To appear.

[23] M. Oliviera, B. Bowen, R. McKenna, and Y.-S. Chang. Fast digital image inpainting. In Proc. of Intl Conf. on Visualization, Imaging and Image Processing (VIIP), page 261266, 2001.

[24] Minqin Wang1,2 Guoqiang Han1 Yongqiu Tu1 Guohua Chen1 Yuefang Gao1s, "TV-Based Texture Image Inpainting", 2008 International Conference on Multimedia and Information Technology.

[25] Paul Ardis and Amit Singhal. Visual salience metrics for image inpainting. Proceedings of the SPIE, 7257, 2009.

[26] Shengfeng Li, Rujing Wang, "Exemplar Image Inpainting by Means of Curvature-Driven Method", 2012 International Conference on Computer Science and Electronics Engineering.

[27] T.F. Chan and S.H. Kang. Error analysis for image inpainting. Journal of Mathematical imaging and Vision, 26(1-2):85\{103, November 2006.

[28] W. Zhang, S.-C. Cheung, and M. Chen. Hiding privacy information in video surveillance system. In Proceedings of the 12th IEEE International Conference on Image Processing (ICIP), volume 3, pages $868\{871$, Genova, Italy, September 2005.

[29] www.wikipedia.com

[30] Y. T. Jia, S. M. Hu, and R. R. Martin. Video completion using tracking and fragment merging. In Proceedings of Pacic Graphics, volume 21, pages $601\{610,2005$.

[31] Yining Deng and b. s. Manjunath. Unsupervised segmentation of color-texture regions in images and video. IEEE Transaction on Pattern Analysis and Machine Intelligence (PAMI), 23(8):800 \{810, 2001.

[32] Yunjun Zhang, Jiangjian Xiao, and Mubarak Shah. Motion layer based object removal in videos. In Proceedings of the Seventh IEEE Workshops on Application of Computer Vision, volume 1, pages $516\{521,2005$. 